\title{
NOD2, an intracellular innate immune sensor involved in host defense and Crohn's disease
}

\author{
W Strober ${ }^{1}$ and $\mathrm{T}$ Watanabe ${ }^{2}$
}

\begin{abstract}
Nucleotide-binding oligomerization domain 2 (NOD2) is an intracellular sensor for small peptides derived from the bacterial cell wall component, peptidoglycan. Recent studies have uncovered unexpected functions of NOD2 in innate immune responses such as induction of type I interferon and facilitation of autophagy; moreover, they have disclosed extensive cross-talk between NOD2 and Toll-like receptors, which has an indispensable role both in host defense against microbial infection and in the development of autoimmunity. Of particular interest, polymorphisms of CARD15 encoding NOD2 are associated with Crohn's disease and other autoimmune states such as graft vs. host disease. In this review, we summarize recent findings regarding normal functions of NOD2 and discuss the mechanisms by which NOD2 polymorphisms associated with Crohn's disease lead to intestinal inflammation.
\end{abstract}

\section{INTRODUCTION}

NOD2 (nucleotide-binding oligomerization domain 2) is a member of the NLR (NOD, leucine-rich repeat (LRR)-containing protein) family of intracellular sensors of pathogen/microbe-associated molecular patterns. ${ }^{1,2}$ It is encoded by the CARD15 gene (on chromosome 16 in humans) and recognizes muramyl dipeptide (MDP), a component of the peptidoglycan present in the bacterial cell wall. Since its discovery in 2000, numerous studies have appeared documenting the role of NOD2 as a key pattern-recognition receptor in innate immune responses. However, its prominence among such pattern-recognition receptors also derives from the fact that polymorphisms in the LRR region of CARD15 are the single most important genetic risk factors for the occurrence of Crohn's disease. ${ }^{1,3}$ In addition, the same polymorphisms increase the risk for graft vs. host diseases (GvHD) in allogeneic bone marrow transplantation, and mutations in the NOD region of CARD15 are causative of Blau's syndrome, a granulomatous inflammation affecting the eyes, skin, and joints. ${ }^{4,5}$ NOD2 function is multifaceted and complex reflecting its interaction with an array of intracellular signaling elements; in this review, we present an analysis of this function using its role in Crohn's disease as a prism for understanding how an innate immune element can be both a host defense factor and a factor implicated in autoimmunity.

\section{THE STRUCTURE OF NOD2}

Like several of the NLR family of proteins, NOD2 contains a NOD domain that is linked on its C-terminal side to a LRR domain and on its N-terminal side to two tandem caspase recruitment (CARD) domains., ${ }^{1,2}$ The LRR domain is the microbe-associated molecular pattern (in this case MDP) recognition region and has a molecular structure similar to the LRR domains found in Toll-like receptors (TLRs). It is thus likely that the polymorphisms in this region associated with Crohn's disease affect the recognition of MDP. The CARD domains are the regions responsible for interactions between NOD2 and "downstream" signaling molecules and thus undergo structural changes upon activation of NOD2 by MDP or other ligands; such structural alterations then facilitate downstream interactions. Finally, the NOD domain (also called the NACHT domain) is involved in the oligomerization of NOD2 that occurs upon its activation and is necessary for CARD region changes allowing NOD2 signaling. Thus, mutations in this region in Blau's syndrome affect the activation of NOD2, rather than the recognition of MDP.

\section{RICK-DEPENDENT NOD2 SIGNALING}

Initial studies of NOD2 function identified the fact that MDP stimulation of NOD2 results in nuclear factor $-\kappa \mathrm{B}(\mathrm{NF}-\kappa \mathrm{B})$ and mitogen-activated protein kinase activation, which then lead to the synthesis of various proinflammatory cytokines and/or chemokines $^{1}$ (Figure 1). However, such activation was not nearly as robust as that obtained with TLR signaling. The major signaling pathway through which MDP-activated NOD2 leads to $\mathrm{NF}-\kappa \mathrm{B}$ activation involves first a conformational change

\footnotetext{
${ }^{1}$ Laboratory of Host Defenses, Mucosal Immunity Section, National Institute of Allergy and Infectious Diseases, National Institutes of Health, Bethesda, Maryland, USA.

${ }^{2}$ Department of Gastroenterology, Kyoto University, Kyoto, Japan. Correspondence: W Strober (Wstrober@niaid.nih.gov) 


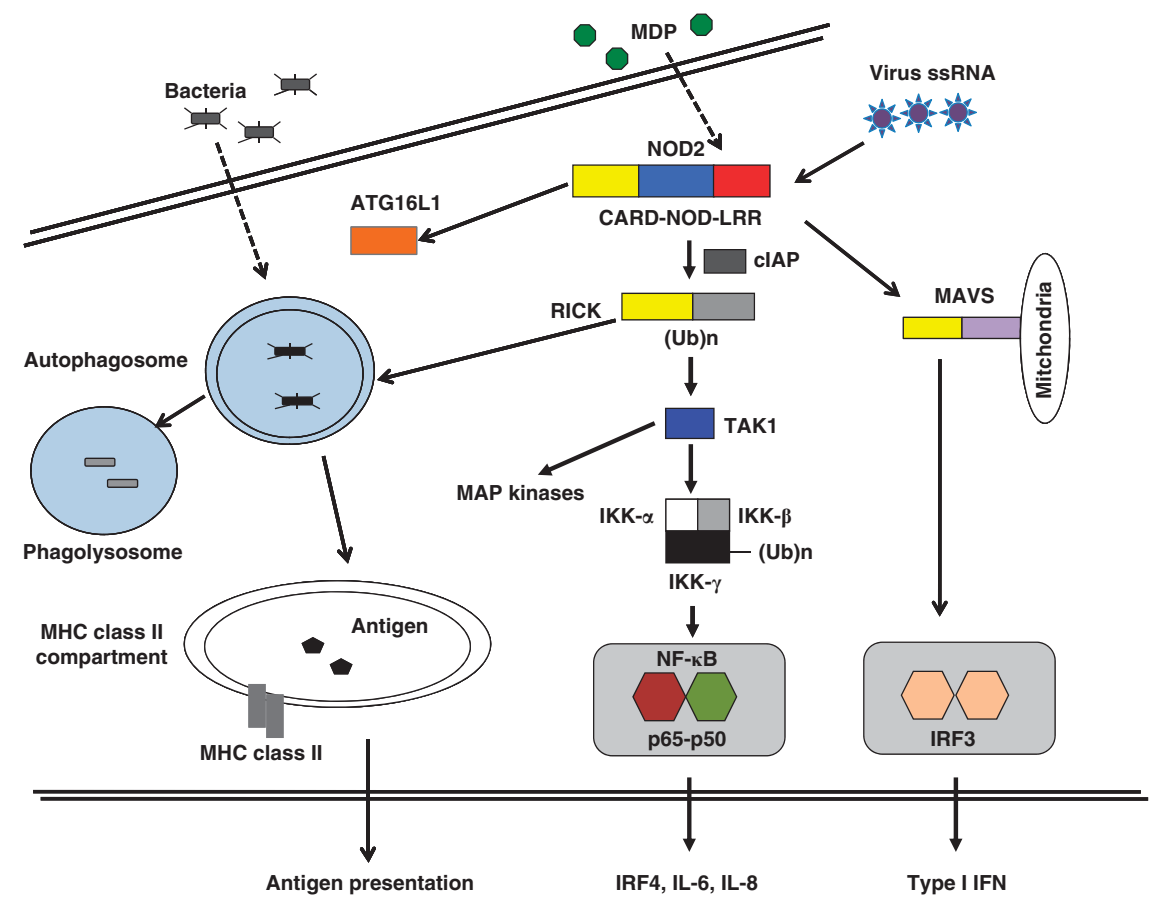

Figure 1 NOD2-mediated signaling pathways. This figure provides an overview of the major signaling pathways of NOD2. The central and perhaps dominant pathway is the RICK-dependent pathway that involves activation of RICK, followed by downstream activation of TAK1; this then leads to activation of MAP kinases and NF-KB. As indicated in the text, interaction of NOD2 with CARD9 or Bid may determine which downstream components are activated. On the right side of the figure, a RICK-independent NOD2 signaling pathway initiated by viral ssRNA is depicted; this involves binding of ssRNA to NOD2 followed by interaction with MAVS, an adaptor protein associated with mitochondria. This leads to activation of IRF3 and induction of type I interferon. On the left side of the figure, interaction of NOD2 with the autophagy proteins in depicted. Such interaction can take the form of RICK-independent interaction with ATG16L1 and trafficking of the latter to the cell membrane, or RICK-dependent (and therefore NF- $\kappa$ B-dependent activation of autophagy proteins. Through this pathway, NOD2 can affect bacterial handling and elimination or antigen presentation. IKK- $\gamma$, inhibitor of nuclear factor kappa-B kinase-gamma; MAP, mitogen-activated protein; MAVS, mitochondria anti-virus signaling

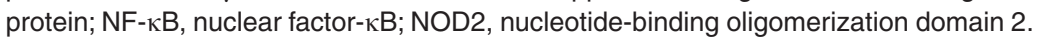

in NOD2 structure, which results in an unfolding of the molecule, followed by oligomerization and exposure of the CARD domain. ${ }^{2}$ This, in turn, leads to NOD2 binding to a downstream adaptor molecule known as RICK (or RIP2) through a CARD-CARD interaction involving the respective CARD domains of NOD2 and RICK. This interaction is critical to subsequent NOD2 induction of cytokine responses after MDP stimulation as mice lacking RICK lack such responses. ${ }^{6,7}$

Studies conducted in HEK cells overexpressing NOD2 and RICK provide evidence that upon close association of RICK with NOD2 (even in the absence of the CARD region), RICK undergoes lysine 63 (K63)-linked polyubiquitination at lysine 209 , which is located at the kinase domain of the molecule. ${ }^{8-10}$ Such polyubiquitination requires the activity of an E3 ligase, and recent studies have suggested that various IAP proteins fulfill this function, as does one of the TRAF proteins (most likely TRAF2) and ITCH, a HECT domain-containing ligase. ${ }^{11}$ Polyubiquitinated RICK then interacts with NEMO (IKK- $\gamma$; inhibitor of nuclear factor kappa-B kinase-gamma ), the NF- $\kappa B$ scaffolding protein the ubiquitination of which initiates NF- $\mathrm{\kappa B}$ activation. ${ }^{9}$ It should be noted that polyubiquitinated RICK also recruits the TAK1 complex (TAK1-TAB1/TAB2), which is essential for RICK-mediated NF- $\mathrm{KB}$ activation and mitogen-activated protein kinase activation. ${ }^{8} 12$ The pattern of polyubiquitination of RICK after NOD2 activation may to some extent determine its precise function. This view is supported by the fact that whereas ITCH-mediated polyubiquitination leads to mitogen-activated protein kinase activation and not NF- $\mathrm{KB}$ activation, polyubiquitination by other $\mathrm{E} 3$ ligases has the reciprocal effect. ${ }^{13}$ This ability of ITCH to divert NOD2 signaling away from NF- $\kappa B$ activation may explain the fact that ITCH depletion is characterized by spontaneous inflammation, particularly at mucosal surfaces.

RICK was originally considered a threonine-serine kinase the kinase activity of which was not necessary for RICK activation of NF- $\mathrm{KB}$. More recently, however, it has been shown to be a tyrosine kinase that causes autophosphorylation at Y474 upon NOD2 activation. ${ }^{14}$ Although such autophosphorylation was also shown to be necessary for optimal NF- $\mathrm{KB}$ activation, it is remains unclear how it contributes to the signaling process. One possibility is that it determines the stability of RICK as it has been shown that cells expressing "kinase-dead" RIP exhibit low RIP expression levels. ${ }^{15,16}$ In unstimulated macrophage cell lines, RICK binds to a mitogen-activated protein-3 kinase component, MEKK4, but dissociates from this complex upon MDP stimulation, thus facilitating the NOD2 signal cascade. However, gene silencing of MEKK4 after MDP stimulation leads to enhanced $\mathrm{NF}-\kappa \mathrm{B}$ activation, suggesting that MEKK4 continues to compete with NOD2 for RICK. ${ }^{17}$ In addition, downregulation of MEKK4 under these conditions leads to reduced TLR2 and TLR4 signaling suggesting that increased levels of RICK have 
downregulatory effects on TLR pathways in accordance with the fact that excess NOD2 also has this outcome (see further discussion below).

A final note relating to RICK-dependent NOD2 signaling concerns its cellular location. Although NOD2 is often referred to as a cytoplasmic sensor of muropeptides, it has in fact been shown that NOD2 is found both in the cytoplasm and in association with the cell membrane. ${ }^{18}$ The latter fact raises the question of whether cell membrane localization is necessary for NOD2 recognition of MDP and the subsequent MDP-induced signaling. Relevant to this issue is the fact that cell membrane association of NOD2 occurs through two amino acids in the C-terminal (LRR) domain harboring the MDP recognition site, and mutations in this site affecting these amino acids prevent membrane association. ${ }^{18}$ In addition, cells expressing NOD2 with such mutations fail to activate NF- $\kappa B$ and induce chemokine production upon stimulation with MDP. These findings support the idea that membrane localization is required for NOD2 activation and fit with the supposition that such localization somehow facilitates the interaction of extracellular NOD2 ligand with its intracellular NLR target molecule. Nevertheless, it remains possible that mutations in NOD2 affecting MDP recognition secondarily impair localization of NOD2 to the membrane by prevention of ligand-induced mechanisms necessary for such localization. This latter view is supported by the finding that downregulation of Rac1GTPase/ $\beta$-PIX, which associates with NOD2 upon activation of the latter, leads to the loss of membrane localization ${ }^{19}$ (see further discussion below). If this alternative idea holds true, it is possible that membrane localization may facilitate negative regulation of NOD2 rather than its initial interaction with ligand and stimulation of NOD2. Finally, it should be noted that the frameshift mutation in the LRR region of NOD2 associated with Crohn's disease leads to loss of the cell membrane-localizing LRR site, whereas other mutations associated with Crohn's disease (R702W and G908R) do not lead to such a loss of localization. ${ }^{18}$ This means that membrane localization is necessary but not sufficient for NOD2 stimulation.

\section{RICK-INDEPENDENT NOD2 ACTIVATION}

Recent studies have provided evidence that MDP-induced, RICK-dependent NOD2 signaling is not the only mechanism by which NOD2 responds to environment pathogen-associated molecular patterns (Figure 1). Most notably, it has been shown that NOD2 responds in vitro to viral ssRNA or in vivo to viral infection with viruses that express ssRNA such as respiratory syncytial virus with IRF3 activation. ${ }^{20}$ This response involves the binding of ssRNA to NOD2, followed by translocation of NOD2 to the mitochondria and interaction with MAVS (mitochondria anti-virus signaling protein), the mitochondrial adaptor molecule previously shown to be involved in viral signaling induced by RIG-I and MDA-5. However, the CARD region of NOD2 is not involved in such an interaction as it is in the case of RIG-I and MDA-5. Finally, the MAVS leads to activation of downstream signaling molecules which lead, in turn, to the production of interferon- $\beta$ and NF- $\kappa B$ activation. Overall, this pathway of NOD2 activation is vastly different from the MDP-induced pathway in that it involves a different ligand and a distinct downstream signaling pathway. Its closest cognate is the pathway used by NOD1, which has also been shown to induce the production of interferon- $\beta{ }^{21}$ In this case, however, NOD1 is responding to a peptidoglycan-derived peptide rather than a virus and acts through a RICK interaction with TRAF3.

\section{BINDING PARTNERS OF NOD2 ANDTHEIR EFFECTS ON NOD2 FUNCTION}

NOD2 binds (directly or indirectly) to an array of intracellular proteins, which either inhibit or enhance its function. Erbin, an LRR-containing protein that is localized to the cell membrane is perhaps the most important of the inhibitory factors. ${ }^{22}$ In co-immunoprecipitation studies, erbin binds to NOD2 through an interaction involving the erbin LRR and the NOD2 CARD domains. Importantly, downregulation of erbin leads to enhanced MDP-induced NOD2 induction of NF- $\kappa B$, indicating that erbin is involved in the negative regulation of NOD2. ${ }^{22}$ Other proteins that bind to NOD2 and that have negative effects on NOD2 function (NF- $\mathrm{BB}$ activation or cytokine induction) include the GTPase-activating protein Centaurinb1 ${ }^{23}$ the angio-associated migratory cell protein, ${ }^{24}$ and the Rac1GTPase, ${ }^{19}$ the latter acting in association with $\beta$-PIX (p21activated kinase-interacting exchange factor), a factor that activates Rac1. The negative regulatory effect of Rac1/ $\beta$-PIX may be linked to the localization of NOD2 to the membrane and its association with erbin, as Racl is known to be involved in actin rearrangement and intracellular traffic; in addition, it has been shown that disruption of the actin cytoskeleton by cytochala$\sin \mathrm{D}$ or a dominant-negative form of Rac1 leads to enhanced NOD2 activity, suggesting that factors that prevent membrane localization minimize NOD2 regulation by erbin.

NOD2 also binds to a number of regulatory factors, which have positive effects on NOD2 function. The first of these to be described, GRIM-19, is a protein involved in cell survival that was shown to have a positive effect on NOD2 function by the fact that downregulation of this molecule in vitro with GRIM19-specific small-interfering RNA leads to decreased NOD2 activation in a luciferase assay system and increased susceptibility of epithelial cells to invasion by Salmonella. CARD9, a second NOD2-binding protein having a positive effect, is a CARD-containing adaptor molecule that binds to NOD2 and RICK through its C-terminal coiled-coil domain and synergizes with NOD2 in response to intracellular infection. ${ }^{25}$

Recently, Yeretssian et al. ${ }^{26}$ used a genome-wide small-interfering RNA interference technique to identify additional proteins binding to NODs and involved in NOD function. By analyzing the data obtained with a multifactored proteinomics approach, they created an extensive probabalistic map of NOD interactions and showed that both NOD1 and NOD2 engage in cross-talk with apoptosis proteins through an interaction with the BCL2 family member, Bid. This led to further studies with Bid-deficient mice, which disclosed that Bid is required for downstream NOD activation of NF- $\kappa \mathrm{B}$ and both for their proinflammatory function and NOD2-regulatory function (ability to confer protection from colitis; see discussion below). Interestingly, it seems 
that Bid binding to NOD2 leads to ERK and NF- $\kappa B$ activation, whereas CARD 9 binding leads $\mathrm{p} 38$ and c-Jun $\mathrm{N}$-terminal kinase activation but no NF- $\mathrm{kB}$ activation. ${ }^{25}$ This suggests that CARD9 and Bid form distinct NOD2-binding complexes that have different signaling outcomes. There is no evidence at present that NOD proteins affect apoptosis through Bid; however, given the fact that BID is cleaved upon activation of apoptosisassociated speck-like protein containing CARD, a critical component of inflammasome, ${ }^{27} \mathrm{NOD} 2$ activation may be connected to the inflammasome through BID.

Finally, it should be mentioned here that NOD2 may also bind to one or more inflammasome proteins that contain CARD domains such as NLRP1 or NLRP3 and thereby participate in interleukin (IL)- $1 \beta$ secretion; this will be more fully explored below.

\section{NOD2 AND AUTOPHAGY}

A new feature of NOD2 activity arises from recent evidence that suggests that NOD2 has important functions in relation to autophagy (Figure 1). Autophagy is a complex intracellular protein degradation mechanism by which the cell forms double-membrane vacuoles that ultimately fuse with lysosomes to eliminate proteins arising from cellular stress responses. ${ }^{28}$ This process also participates in the breakdown of ingested pathogenic bacteria and in the processing of antigens for antigen presentation in immune responses; hence, it also has a role in immunological responses and host defense.

In one study of the relation of NOD proteins to autophagy, it was shown that NOD-deficient (NOD1- or NOD2-deficient) cells transduced with NOD constructs manifest autophagy upon exposure to NOD ligands ${ }^{29}$ however, autophagy also occurs with lipopolysaccharide (LPS) stimulation in NOD2-deficient cells indicating that NOD2 deficiency can be compensated by other innate responses. In further studies, these authors linked the role of NOD proteins in autophagy to the ability of these proteins to bind to ATG16L1 a key protein in the autophagy signaling pathway that also exhibits polymorphisms associated with Crohn's disease. ${ }^{3}$ The authors provided evidence that NOD proteins bind to ATG16L1 and co-localize with NOD2 at the plasma membrane. Thus, they postulated that NOD2 recruits ATG16L1 to the membrane and thus facilitates the formation of an autophagosome around the invading bacterium. ${ }^{29}$ Finally, they showed that dendritic cells from gene-targeted mice with a frameshift NOD2 mutation, which as discussed above does not localize to the membrane, exhibit a reduced autophagy response to Shigella.

A complementary picture emerged in a second study of NOD2 and autophagy. Here, the authors found that NOD2 stimulation with MDP led to autophagic response in common with that induced by either TLR2 or TLR4 ligands. ${ }^{30}$ In knockdown studies, this response was specific for NOD2 and required intact RICK function in contrast to the study of autophagy described above in which RICK-deficient cells still manifested NOD interaction with ATG16L1. In further studies, it was found that NOD2 stimulation induced major histocompatibility complex class II expression to the same extent as a TLR2 ligand, and NOD2 knockdown led to decreased proliferative response to a Salmonella organism. Finally, in studies of the function of NOD2 in patients expressing NOD2 or ATG16L1 polymorphisms associated with Crohn's disease, it was shown that cells containing NOD2 with such polymorphisms failed to express major histocompatibility complex class II and to respond to Salmonella with proliferative responses. In addition, it was shown that cells with such polymorphisms had a decreased capacity to localize adherent-invasive Escherichia coli to lysosomes and to kill such bacteria.

These studies together establish that NOD proteins participate in the induction of autophagy and, as such, have a role in both antigen presentation and bacterial killing. However, they raise several unanswered questions. The first concerns the fact that NOD activation seems to be uniquely important to induction of autophagy as cells containing NOD2 variants expressing Crohn's disease-associated polymorphisms show impaired autophagic responses, even though the same cells express intact TLR2 and TLR4 that are also capable of inducing autophagy. ${ }^{31}$ In one of these studies, this could be explained by the fact that NOD proteins have an essential and non-redundant role in the recruitment of an autophagic protein to the cell membrane. However, this explanation does not account for why NOD1 in cells with such NOD2 polymorphisms does not provide compensatory recruitment function. The second question concerns the fact that whereas in one study, NOD effects on autophagy were independent of RICK signaling, ${ }^{29}$ in the other study, such signaling was essential. ${ }^{30}$ Lack of RICK-mediated NOD signaling seems unlikely because such signaling has been shown to be necessary for membrane localization, and hence recruitment of autophagy proteins to the membrane should not be possible in the absence of signaling (see discussion above). On the other hand, if in fact signaling is necessary, the question arises as to how NOD2 proinflammatory signaling differs from that of TLRs and thus explains the special effects of NOD2 on autophagy-dependent bacterial handling.

\section{NOD2 PARTICIPATION IN INNATE IMMUNE RESPONSES}

Studies in both mice and humans have established that MDP stimulation of NOD2 in antigen-presenting cells results in variable responses, which in most cases are far less than those obtained with TLR stimulation. ${ }^{32}$ To some extent, these different levels of responses may reflect cell type-specific factors such as the aforementioned adaptor molecules that facilitate NOD2 stimulation. ${ }^{1}$ It should be noted, however, that although NOD2 stimulation itself results in weak responses, such stimulation has a considerable capacity to augment TLR responses. ${ }^{33}$ Such augmentation may result from the fact that TLR stimulation upregulates NOD2 expression, and combined stimulation of cells through upregulated NOD2 and a TLR has a synergistic effect similar to that seen for other combinations of TLR stimulants. ${ }^{34}$

If indeed the main effect of NOD2 stimulation is to enhance TLR-mediated inflammatory responses, rather than to induce the latter itself, the question arises as to whether a 2-3-fold enhancement of individual TLR responses can fully explain the well-established host defense role of NOD2 to be discussed 
below, particularly if such enhancement can come from TLR synergy. One way in which NOD2 responses can complement or enhance innate immune response mediated by TLRs or other innate response elements occurs by virtue of its position as an intracellular response factor, which more efficiently addresses the neutralization of pathogens residing in intracellular sites. Evidence that this may be so comes from the aforementioned studies that NOD2 may facilitate autophagy and thus the killing of intracellular organisms through autophagic mechanisms. In addition, it has been shown with DNA microarray technology that host transcriptional responses to vacuolar and cytosolic organisms are fundamentally different: vacuolar organism responses are MyD88 dependent (i.e., TLR dependent), whereas cytosolic organisms responses are IRF3 and NOD2 dependent. ${ }^{35}$ The latter accounts for the fact that cytosolic organisms induce interferon- $\beta$ during infection with Mycobacterium tuberculosis, which has been shown to be an important host defense component during infection with this organism.

Another factor that may account for the way in which NOD2 responses add to TLR responses lies in the fact that NOD2 responses can conceivably provide host defense function in areas of the body where TLR expression is reduced either because the cellular milieu or because TLR function has been compromised by previous exposure to TLR ligands and the induction of TLR tolerance. This could be particularly true in the gastrointestinal (GI) tract where the commensal microflora provide a source of TLR ligands that might render gut epithelial cells or antigen-presenting cells tolerant to TLR ligands. ${ }^{1}$ Relevant to this point, Kim et al. ${ }^{36}$ have shown that cells or mice made tolerant to various TLRs because of previous exposure to TLR ligands exhibit heightened responses to either NOD2 or NOD1 ligands and, more importantly, bacterial clearance of Listeria monocytogenes was dependent on NOD2 and NOD1 if mice had been previously exposed to LPS or E. coli. Thus, in this situation, NOD2 (and NOD1) acted as a second line of defense against pathogenic invasion.

Yet another mechanism by which NOD2 can contribute to host defense in the face of a more potent TLR response is by the induction of particular cytokines through novel mechanisms. This possibility finds support in studies reported by Karin and his associates ${ }^{37}$ who provided evidence that MDPactivated NOD2 binds to the inflammasome protein, NLRP1, and the complex thus formed binds pro-caspase I through the NOD2 and/or the NLRP1 CARD domains. This, in the absence of NOD2 signaling through RICK, then leads to an activated caspase I, which then acts on pro-IL-1 $\beta$ or pro-IL-18 to form mature forms of these cytokines that can then be secreted. The function of this complex was shown in the context of Bacillus anthracis infection in which it was found that $B$. anthracis lethal toxin activation of NLRP1 and the induction of IL- $1 \beta$ required the presence of NOD2. In related studies involving deletion mutants of NOD2, it was shown that unstimulated intact NOD2 maintains an inactive coiled state as a result of interaction between the LRR domain and the CARD domain and that deletion of the LRR domain results in an active uncoiled NOD2 that constitutively binds to NLRP1 and initiates IL- $1 \beta$ secretion.
Furthermore, NOD2 with a Crohn's-like frameshift mutation in the LRR acts like an uncoiled NOD2 LRR deletion mutant and spontaneously interacts with NLRP1 to induce IL- $1 \beta$ secretion. Whether IL- $1 \beta$ secretion arising from such a mechanism explains the association of NOD2 polymorphisms with Crohn's disease is addressed more fully below.

Other studies relating to NOD2 and inflammasome activity tend to corroborate the above finding, although in these instances, the interaction of NOD2 is generally believed to be with NRLP3 rather than with NRLP1. ${ }^{38,39}$ Regardless of which inflammasome protein is involved, these studies predict that NOD2 stimulation by MDP when coupled with TLR stimulation (in the absence of ATP required for conventional inflammasome activation) would be associated with an early and prominent IL-1 $\beta$ secretory response that exceeds the synergistic response between MDP and TLR ligands for cytokines not related to inflammasome activity; however, this does not seem to be the case. $^{33}$

Although NOD2 usually has a positive role in innate immune responses, there is a growing body of evidence that suggests that NOD2 also exerts regulatory function that inhibits innate responses. The first evidence for this type of NOD2 function came from Watanabe et al., ${ }^{32}$ who showed that NOD2-deficient mice as compared with NOD2-intact mice gave rise to increased IL-12p70 responses when stimulated with peptidoglycan (PGN), a TLR2 stimulant that gives rise to MDP. Furthermore, addition of MDP to PGN-stimulated antigen-presenting cells from NOD2-intact mice led to decreased IL-12p70 responses. These and later more extensive studies in which the effects of MDP pre-treatment on TLR responses of both mouse and human dendritic cells was determined, established that MDP could downregulate TLR2 responses and other TLR responses and that, in mice, such downregulation required the presence of NOD2. Furthermore, they showed that such downregulation affected a broad range of cytokine responses resulting from TLR stimulation. As noted below, these findings of Watanabe et al. were corroborated by a host of related studies and by studies of other investigators. ${ }^{40-42}$ One dissenting voice, however, was that of Kim et al. ${ }^{36}$ who showed that although pre-stimulation with MDP led to diminished subsequent MDP responses it had, if anything, the effect of augmenting subsequent TLR responses. It should be noted, however, that these investigators used far lower pre-stimulatory concentrations of MDP than those used in the studies by Watanabe et al.

In further studies expanding on the above findings relating to NOD2-regulatory function, it was shown that NOD2deficient mice, as compared with NOD2-intact mice, exhibited greatly increased susceptibility to colitis caused by intrarectal administration of recombinant $E$. coli expressing ovalbumin peptide (termed "ECOVA") to mice adoptively transferred ovalbumin peptide-specific T cells. ${ }^{43}$ These findings were supported by those from a series of studies in which the function of NOD2 was probed in mice with increased NOD2 signaling either because they overexpressed NOD2 or because they had been administered exogenous MDP. In NOD2 overexpression studies, it was found that mice carrying a NOD2 transgene in 
major histocompatibility complex class II-positive cells had antigen-presenting cells, which exhibited reduced PGN-induced cytokine responses; in addition, these mice manifested greatly increased resistance to the induction of colitis by intrarectal administration of PGN or by trinitrobenzene sulfonic acid, agents that cause an IL-12-dependent acute colitis. ${ }^{44}$ Similarly, it was found that in mice, administration of an intact NOD2expressing plasmid led to total resistance to induction of trinitrobenzene sulfonic acid colitis, whereas administration of a plasmid expressing a NOD2 with a frameshift mutation similar to that in Crohn's disease led to only a minor increase in resistance to induction of trinitrobenzene sulfonic acid colitis. ${ }^{44}$

Parallel studies in which NOD2 signaling was augmented by administration of exogenous MDP yielded results compatible with those from the overexpression studies. Here, it was observed that parenteral administration of MDP to intact mice led to virtually complete blockade of either dextran sulfate sodium colitis or trinitrobenzene sulfonic acid colitis induction, whereas such administration of MDP to NOD2- or RICK-deficient mice had no effect on dextran sulfate sodium colitis induction. ${ }^{45}$ Moreover, MDP-treated mice contained lamina propria cells manifesting greatly decreased responses to multiple TLR ligands. In a final study along these lines which combined overexpression of NOD2 with MDP administration, NOD2-deficient mice were administered plasmids expressing intact NOD2 or NOD2 with a frameshift mutation. ${ }^{45}$ It was found that mice administered an intact NOD2 vector were protected from dextran sulfate sodium colitis when administered parenteral MDP, whereas mice administered mutated NOD2 or control (empty) vector were not so protected. Thus, provision of intact NOD2 but not mutated NOD2 rendered NOD2-deficient mice capable of responding to the colitis-protective effect of MDP administration. Overall, these studies of mice in which NOD2 signaling was augmented in NOD2-intact mice or simply provided in NOD2-deficient mice provided powerful evidence that NOD2 signaling had a profound tolerogenic effect on TLR responses which could abrogate induction of colitis.

Studies of mice subjected to GvHD in which the effects of NOD2 deficiency in hematopoietic donor and recipient cells could be assessed provided corroboration of the above studies of the regulatory effect of NOD2 signaling in hematopoietic cells. ${ }^{46}$ These studies showed that allogeneic bone marrow transplantation from NOD2-deficient mice into NOD2-intact recipient mice led to increased activation of donor $\mathrm{T}$ cells and therefore more intense GvHD responses. One important implication of these studies is that lack of NOD2-regulatory effects causes not only gut inflammation but also inflammation in sterile organs in which the confounding effects of the gut flora are not operative. In this model, increased inflammation leading to enhanced GvHD occurs within a sterile environment presumably under the influence of endogenous TLR ligands.

The above studies of NOD2 regulation of TLR responses in mice presaged studies in humans relating to this phenomenon. In one such study, it was shown that pre-stimulation of mononuclear cells from normal individuals with MDP or with Salmonella organisms led to reduced LPS-induced tumor necrosis factor responses, but had no effect on IL-6 or IL-10 responses. ${ }^{41}$ In a more comprehensive study, it was shown that pre-stimulation of macrophages from normal individuals had major downregulatory effects on subsequent induction of tumor necrosis factor, IL- 8 , and IL- $1 \beta$ to secondary stimulation of cells with MDP, the TLR4 ligand lipid A, the TLR2 ligand Pam3Cys, or IL-1 $\beta .{ }^{42}$ In this study, the degree of downregulation was increased with the duration of pre-stimulation prompting the authors to refer to the pre-stimulation as chronic stimulation. In a second set of studies, anti-IL- $1 \beta$ addition to the cultures partially reversed downregulation by MDP in some individuals, but the mechanism of such inhibition was not provided. ${ }^{42}$

The mechanism of NOD2 regulation of TLR response discussed above is as yet poorly understood. One important possibility suggested by studies in mice is that NOD2 is dependent on IRF4, a molecule previously shown to disrupt the MyD88-IRF5IRAK1 complex by competing with IRF5 binding to MyD88. ${ }^{47}$ Evidence for involvement of IRF4 comes from the observation that MDP inhibition of dextran sulfate sodium colitis is completely reversed in IRF4-deficient mice. ${ }^{45}$ In human dendritic cells, downregulation of IRF4 by small-interfering RNA led to partial reversal of NOD2 regulation. In addition, in studies in human cells, it has been found that MDP pre-stimulation inhibits IRAK-1 kinase activity, a possible outcome of disruption of the TLR signaling complex by IRF4. It seems possible that activated RICK resulting from NOD2 stimulation binds to IRF4 and the resulting complex enhances the inhibitory capacity of IRF4, but this remains to be demonstrated. Another possible mechanism is that NOD2 regulation depends on upregulation of IRAK-M, an intermediate that has been implicated in LPS tolerance and which inhibits IRAK1 activation. ${ }^{48}$ Indeed, in human macrophages, downregulation of IRAK-M by small-interfering RNA led to partial reversal of NOD2 regulation, but in a similar study of mouse cells this was not seen.$^{41}$ Finally, studies in human cells provided evidence that NOD2 regulation could be blocked at least partially by addition of antibodies to several anti-inflammatory mediators, such as IL-10, transforming growth factor- $\beta$, and IL-1R antagonist and blocked completely by antibodies to all of these mediators. ${ }^{42}$ In addition, it could be blocked by addition of rapamycin and thus by inhibition of the mammalian target of rapamycin signaling pathway. This mechanism of NOD2 regulation is difficult to understand, given the fact that it implies that NOD2 signaling results in a diverse and unlikely range of anti-inflammatory molecules. ${ }^{42}$

\section{HOST DEFENSE FUNCTIONS OF NOD2}

As will become apparent from the discussion below, NOD2 has unequivocal function as a host defense factor not only in relation to bacteria which express peptidoglycan and thus MDP but also in relation to viruses and parasites. However, unlike TLRs that exist on the surface of the cell, NOD2 is an intracellular sensor and thus can mount host defense responses only in relation to organisms that either facilitate entry of MDP into the cell or enter the cell themselves to establish intracellular infection. One possible scenario of NOD2 responsiveness to bacteria is that bacteria shed peptidoglycan fragments including MDP as a 
result of a normal cell wall remodeling process, and the MDP so released is then taken up by endocytosis or by a cell transporter. Whether this proposed mechanism of uptake is influenced by localization of NOD2 to the cell membrane is unclear. Cellular uptake can also conceivably occur through cell membrane pores created by pore-forming toxins or by injection of PGN fragments into the cell through type IV secretion systems.

Studies to demonstrate the specific contribution of NOD2 to host defense have relied mainly on observations made in NOD2-deficient mice. The first study of the role of NOD2 in host defense in NOD2-deficient mice involved responses to the intracellular pathogen L. monocytogenes in which it was shown that oral but not parenteral administration of the organism led to more hepatic infection in the NOD2-deficient mouse than in control mice. ${ }^{6}$ This increased susceptibility to infection was associated with defective killing of bacteria by intestinal crypt cells and deficient production of cryptins. The latter are NOD2-dependent anti-bacterial peptides produced in specialized epithelial cells in the crypts of the terminal small intestine called Paneth cells, which have been shown to manifest a much higher level of NOD2 expression than other epithelial cells. ${ }^{49}$ The idea that NOD2 host defense in the intestine is related to a large extent on its ability to induce $\alpha$-defensin production was subsequently more firmly supported by studies showing that Helicobacter hepaticus infection of NOD2-deficient mice but not of normal mice causes a Th1 cytokine-associated granulomatous infection in the terminal ileum and that this reaction is prevented in NOD2-deficient mice that carry a transgene that expresses a cryptin ( $\alpha$-defensin 5 ) in Paneth cells (and in other cells). ${ }^{50}$

The above study showing that NOD2 deficiency had no effect on parenteral L. monocytogenes infection suggested that the role of NOD2 in host defense is limited to the infection of the GI tract. However, several studies using NOD2-deficient mice suggest that NOD2 contributes to host defense against Staphylococcus aureus infection of the lung and skin, ${ }^{51}$ Chlamydophila pneumoniae of the lung, ${ }^{52}$ and systemic Toxoplasma gondii infection. ${ }^{53}$ In the latter case, NOD2 deficiency in T cells rather than dendritic cells were shown to be the cause of the increased severity of infection as discussed more extensively above. ${ }^{53}$ Most importantly perhaps, evidence has accumulated suggesting that NOD2 may be particularly important as a host defense factor in $M$. tuberculosis infection. First, in in vitro studies, it was shown that only M. tuberculosis and related bacteria induced tumor necrosis factor- $\alpha$ responses that were NOD2 dependent, perhaps because of the fact that $M$. tuberculosis produces an $\mathrm{N}$-glycolylated form of MDP that has an increased propensity to stimulate NOD2 (as compared with the N-acetylated form produced by most bacteria) and which thus might allow $M$. tuberculosis organisms to elicit a quantitatively and qualitatively superior NOD2 host defense response. ${ }^{54,55}$ With respect to the latter, it was shown that phagocytosis of $M$. tuberculosis results in the induction of type I interferon production through a unique pathway involving the RICK, TBK1, and IRF5 pathways. ${ }^{56}$ Second, NOD2 deficiency has been shown in two independent studies to be associated with decreased cytokine responses to
M. tuberculosis infection..$^{57,58}$ However, although in one study, this was associated with increased bacterial burden and decreased survival, ${ }^{57}$ in another study, this was not associated with differences in these parameters. ${ }^{58}$ This latter study thus introduces an element of uncertainly regarding the overall role of NOD2 in M. tuberculosis infection.

In the studies cited above documenting that NOD2 serves as a host defense element in various infections, the infections studied were by and large acute infections testing the contribution of NOD2 to early responses. However, another picture may emerge when more chronic infections are studied. This is well illustrated in the case of infection with Borrelia burgdorferi, the cause of Lyme's disease. In this case, although in vitro studies of NOD2-deficient macrophages revealed decreased cytokine responses to B. burgdorferi, in vivo studies of NOD2-deficient mice revealed increased inflammatory manifestations of infection such as increased arthritis and carditis. ${ }^{59}$ Importantly, the latter could be tied to the fact that more prolonged stimulation of NOD2-deficient macrophages led to increased rather than decreased cytokine responses. The authors thus concluded that NOD2 responses are biphasic: early responses are positive, whereas latter responses are negative.

As mentioned above in a different context, NOD2 participates in host defense against viruses that express ssRNA such as respiratory syncytial virus through a RICK-independent mitochondrial mechanism using MAVS. In addition, there is evidence that it also has a anti-dsRNA viral host defense role by binding to $2^{\prime}-5^{\prime}$-oligo adenylate synthetase and causing activation of RNase-L through a RICK-dependent pathway. ${ }^{60}$

Finally, NOD2 also has a role in host defense by influencing adaptive immune responses. Here, it is interesting to recall that MDP was shown very early to have considerable adjuvant properties with respect to induction of antibody responses. This can now be attributed to MDP activation of NOD2 as studies of various analogs of MDP reveal that adjuvanticity and the ability to stimulate NOD2 are highly correlated. However, just how MDP positively affects antibody responses is still unclear. One possibility is that NOD2 stimulation leads to increased production of various cytokines involved in helper T-cell responses and indeed there is evidence from one study that NOD2 stimulation of mouse cells leads to an antigen-specific Th2 response, which is known to favor antibody production. ${ }^{61}$ However, in other situations, NOD2 stimulation leads to Th1 or Th17 responses so that a specific NOD2-associated cytokine production pattern has not been established. ${ }^{62,63}$ Another possibility is that NOD2 induces cytokines such as BAFF, which has a specific effect on $\mathrm{B}$-cell survival and expansion; the fact that BAFF is an NF- $\mathrm{kB}$ dependent cytokine favors this view. However, TLRs would have a similar positive effect on BAFF induction, so that this is not likely to be a NOD2-specific mechanism.

\section{NOD2 AND CROHN'S DISEASE}

Genome-wide association studies have identified 30-40 distinct genetic polymorphisms associated with Crohn's disease. Of these, polymorphisms affecting the CARD15 gene on chromosome 16 (originally identified as the IBD1 locus before 
genome-wide association studies) confers by far the greatest risk for disease: in individuals of European ancestry, homozygous or compound heterozygous carriers of the polymorphisms have a 17.1-fold increased risk of developing disease and heterozygous carriers have a 2.4-fold increased risk. ${ }^{3}$ Thus, NOD2 function has a relatively powerful effect on disease pathogenesis, and the discovery of how it mediates this effect is likely to provide important insights into the cause of Crohn's disease.

The consensus view of the cause of Crohn's disease is that it is due to an excessive response to components of the normal gut microflora. This excessive response can arise from an abnormality affecting the intrinsic reactivity of the mucosal immune system to mucosal antigens or, more simply, to increased exposure of an essentially normal mucosal immune system to microflora components resulting from increased exposure of mucosal immune system to lumenal bacteria. ${ }^{64}$ Within the context of this latter possibility, it is reasonable to suggest that a NOD2 polymorphism causes an increased risk for $\mathrm{CD}$ because the polymorphisms lead to decreased MDP-induced cytokine responses that are critical for the control of potentially inflammation-inducing commensal microbiota in the lumen of the gut. ${ }^{1}$

A number of findings can be marshaled in support of this theory. First, numerous in vitro and in vivo studies of MDPinduced responses of cells of patients bearing $\mathrm{CD}$-associated NOD2 polymorphisms document the fact that the presence of these polymorphisms lead to reduced MDP-induced proinflammatory responses both at the molecular level (e.g., reduced activation of NF- $\mathrm{KB}$ ) and at the cellular level (e.g., reduced production of proinflammatory cytokines. ${ }^{1}$ This decreased function is not unexpected as the LRR region is the MDP-sensing domain and the polymorphism is likely to be associated with impaired MDP recognition. Second, as discussed above, studies of NOD2-deficient mice have disclosed that the absence of NOD2 leads to increased susceptibility to GI infections with L. monocytogenes or $H$. hepaticus and that such infection is attributable to defective NOD2-induced cryptin production by small intestinal Paneth cells. ${ }^{6,50}$ These findings thus suggest that NOD2 dysfunction results in qualitative or quantitative changes in the commensal organisms present in the terminal ileum which, in turn, leads to entry of such organisms into the lamina propria through Peyer's patches and initiation of Crohn's-like inflammation. This scenario is favored by evidence that patients with NOD2 abnormalities manifest more severe defects in $\alpha$-defensin production than is normally seen in Crohn's disease and NOD2 polymorphisms are particularly associated with Crohn's disease affecting the terminal ileum ${ }^{65}$ (Figure 2).

A third and final support of the possibility that NOD2 polymorphisms lead to Crohn's disease susceptibility because of compromised ability to control gut commensal organisms come from recent work, already discussed above, showing that NOD2 is involved in autophagy and thus in the trafficking of ingested organisms to lysosomes where they are eliminated. In one study, evidence was presented showing that cells from patients with Crohn's disease-associated NOD2 abnormalities have decreased autophagic responses to infection and defective killing of ingested pathogenic bacteria; this was associated with defective NOD2 signaling function which led to impaired autophagy in spite of the fact that LPS-induced (TLR4-mediated) autophagy induction was presumably intact. ${ }^{30}$ In a second study, ${ }^{29}$ it was shown that cells from patients with Crohn's disease-associated NOD2 abnormalities fail to manifest a unique NOD2 function not dependent on NOD2 signaling, namely the binding to a key autophagy protein, ATG16L1 and transporting the latter to the cell membrane where it initiates autophagy upon interaction with entering bacteria. The implication of both studies is that defective NOD2 function results in deficient autophagic responses to bacteria and therefore uncontrolled bacterial expansion.

Although the above support for the conclusion that NOD2 abnormalities lead to Crohn's disease because they, in effect, cause a kind of mucosal immunodeficiency that impairs the ability of the mucosal immune system to exclude organisms from the lamina propria is initially compelling, other considerations suggest that this conclusion is deeply flawed. The fact is that these studies point to a defect in the handling of mucosal pathogens, yet there is no evidence that Crohn's disease patients are more susceptible to infection with such agents or have a high frequency of such infection, even when they are treated with agents that tend to undermine immune function. In addition, there is little evidence that Crohn's disease is preceded by the presence of increased numbers of bacteria in the lamina propria. ${ }^{66}$ Finally, it cannot be said that the necessarily small numbers of commensal organisms that persist in the lamina propria as a result of a NOD2 abnormality would necessarily cause inflammation. Recent studies have shown that intentional impairment of colonic permeability elicits a regulatory $\mathrm{T}$-cell response that turns off inflammation. ${ }^{67}$

Also bearing on the question of whether NOD2 abnormalities lead to immunodeficiency are on-going studies suggesting that Crohn's disease may be due to colitogenic organisms rather than by non-inflammatory gut commensals. Such organisms may be more likely to gain a foothold in the lamina propria in patients with defective NOD2 function. The most creditable data relating to this question derive from studies showing that a "semi-pathogenic" E. coli (called adherent-invasive E. coli) can be cultured from the terminal ileum of a substantial portion of patients with Crohn's disease, which has attributes that suggest it may cause Crohn's disease. ${ }^{68}$ Among these is the ability of these organisms to persist in macrophages after ingestion and to induce macrophages to produce tumor necrosis factor- $\alpha$; in addition, these organisms contains fimbriae that enable it to bind to $\mathrm{M}$ cells overlying Peyer's patches which then mediate its entry into Peyer's patches. ${ }^{69}$ Despite these attributes, adherent-invasive E. coli is not likely to be a primary factor in Crohn's disease as there is evidence that its accumulation in the small intestine is a secondary event related to the appearance of epithelial cell-binding sites induced by cytokines associated with a pre-existing inflammatory response. ${ }^{69}$ Perhaps more relevant to the present discussion is the fact that mice lacking NOD2 express increased amount of the $\mathrm{M}$ cell-associated binding protein capable of mediating bacterial entry in to Peyer's patches, yet do not develop colitis 


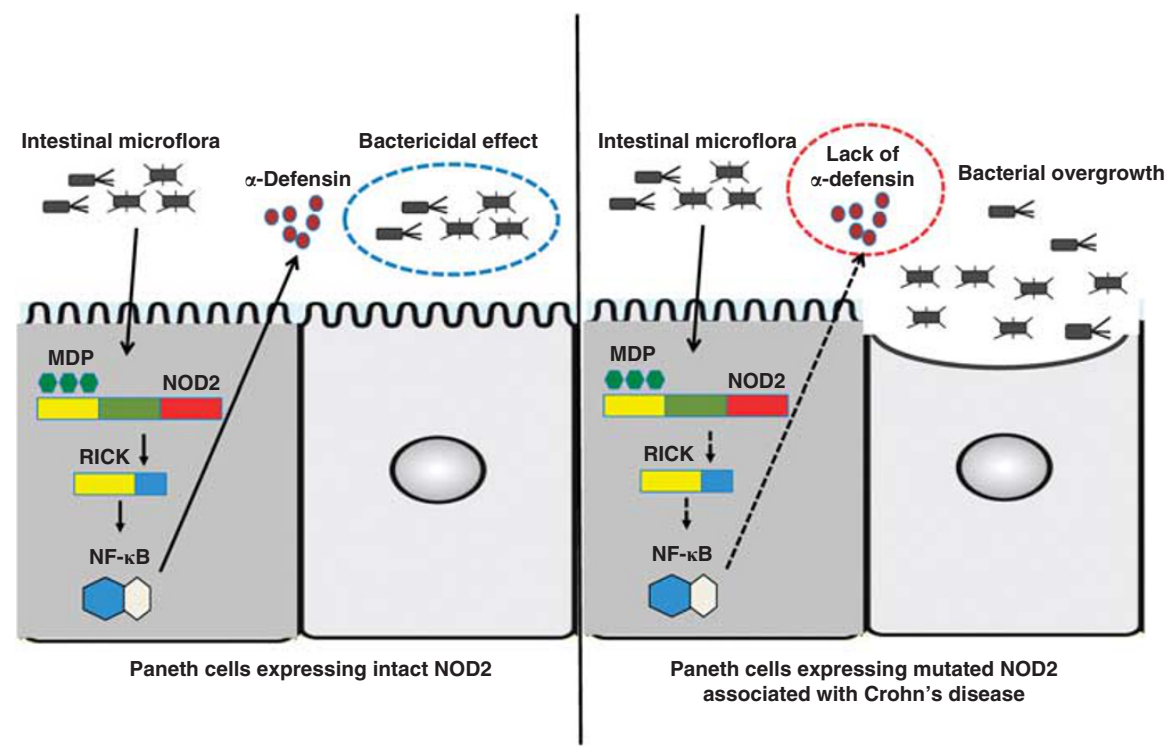

Figure 2 Impaired production of $\alpha$-defensin by Paneth cells in the presence of Crohn's disease-associated NOD2 mutations. MDP derived from intestinal microflora activates NOD2 expressed by Paneth cells to induce $\alpha$-defensin production. $\alpha$-Defensins exert bactericidal effects against intestinal microflora to control the concentration of the latter in the terminal ileum. In the presence of Crohn's disease-associated NOD2 polymorphisms, $\alpha$-defensin production by Paneth cells may be reduced, leading to increased microflora concentration in the small intestine. This, coupled with other effects of abnormal NOD2 function can lead to small intestinal Crohn's inflammation. MDP, muramyl dipeptide; NF-kB, nuclear factor-кB; NOD2, nucleotide-binding oligomerization domain 2.

when infected with adherent-invasive E. coli. ${ }^{69}$ Finally, the same or similar argument applies to other possible colitogenic agents that have been recently identified in that these too are likely to be secondary rather than primary disease factors. ${ }^{70}$

The possible role of NOD2 acting through a defect in Paneth cell $\alpha$-defensin production as a cause of Crohn's disease has some creditability, given evidence in mice and humans regarding $\alpha$-defensin function in the terminal ileum described above. However, this role is mitigated by the fact that substantial decreases in $\alpha$-defensin occurs in Crohn's disease patients without NOD2 abnormalities and evidence for excessive decreases has only been found in patients with the NOD2 polymorphism causing a frameshift ( $50 \%$ of patients with polymorphisms) ${ }^{71}$ In addition, evidence that excessive decrease occurs even in the latter patients is controversial. ${ }^{72}$ The most conservative conclusion with regard to NOD2 and $\alpha$-defensin is that it leads in some cases to increased numbers of commensal organisms in a part of the GI tract that normally contains relatively few organisms (compared with the colon) and therefore leads to small intestinal disease in individuals who have a tendency to develop Crohn's disease because of NOD2 effects on other aspects of mucosal immune function (Figure 2).

Two other theories of how NOD2 polymorphisms relate to Crohn's disease relating to IL- 10 and IL- $1 \beta$ production can be dispensed with rather easily. In one study involving IL-10, evidence was presented suggesting that NOD2 exerts a positive effect on IL-10 production and thus NOD2 polymorphisms lead to impaired production of this anti-inflammatory cytokine. ${ }^{73}$ The problem with this idea is that although NOD2 deficiency may lead to reduced IL-10 responses, this reduction is accompanied by similar decreases in proinflammatory responses so that one cannot say that NOD2 preferentially impairs regulatory IL-10 responses. In another study involving IL-1 $\beta$, it was shown that gene-targeted mice bearing the Crohn's disease frameshift mutation have excessive proinflammatory IL-1 $\beta$ responses. ${ }^{74}$ However, it is not clear that these mice mimic NOD2 abnormalities in patients and it has been shown repeatedly that the NOD2 frameshift abnormality is, associated with decreased rather than increased IL- $1 \beta$ responses. ${ }^{75}$

A final theory as to how NOD2 polymorphisms increase the risk for Crohn's disease relates to the fact that NOD2, in common with other innate signaling elements (TLRs) has the dual capacity to both initiate innate proinflammatory responses and ultimately inhibit such responses. This regulatory function of NOD2 is likely to be particularly important in the GI mucosa where an extensive host microflora, with its rich content of bacterial wall peptidoglycan and other TLR ligands, has the potential to cause continuous stimulation of mucosal immune elements. On this basis, it is reasonable to suggest that defective NOD2 signaling leading to impaired regulation of TLR responses to commensal components, especially TLR2 responses, manifests itself as Crohn's disease (Figure 3).

As discussed above, in experimental systems, the regulatory function of NOD2 was demonstrated by in vitro studies in which it was shown that pre-stimulation of cells with MDP led to diminished subsequent responses to TLR ligands. ${ }^{45}$ In addition, in extensive in vivo studies of mice with enhanced NOD2 signaling either because of NOD2 overexpression in antigen-presenting cells or because of administration of exogenous MDP, proinflammatory responses cytokine responses were greatly reduced and were highly resistant to the induction of experimental colitis. ${ }^{11,26,44,45}$ Finally, in studies directly 


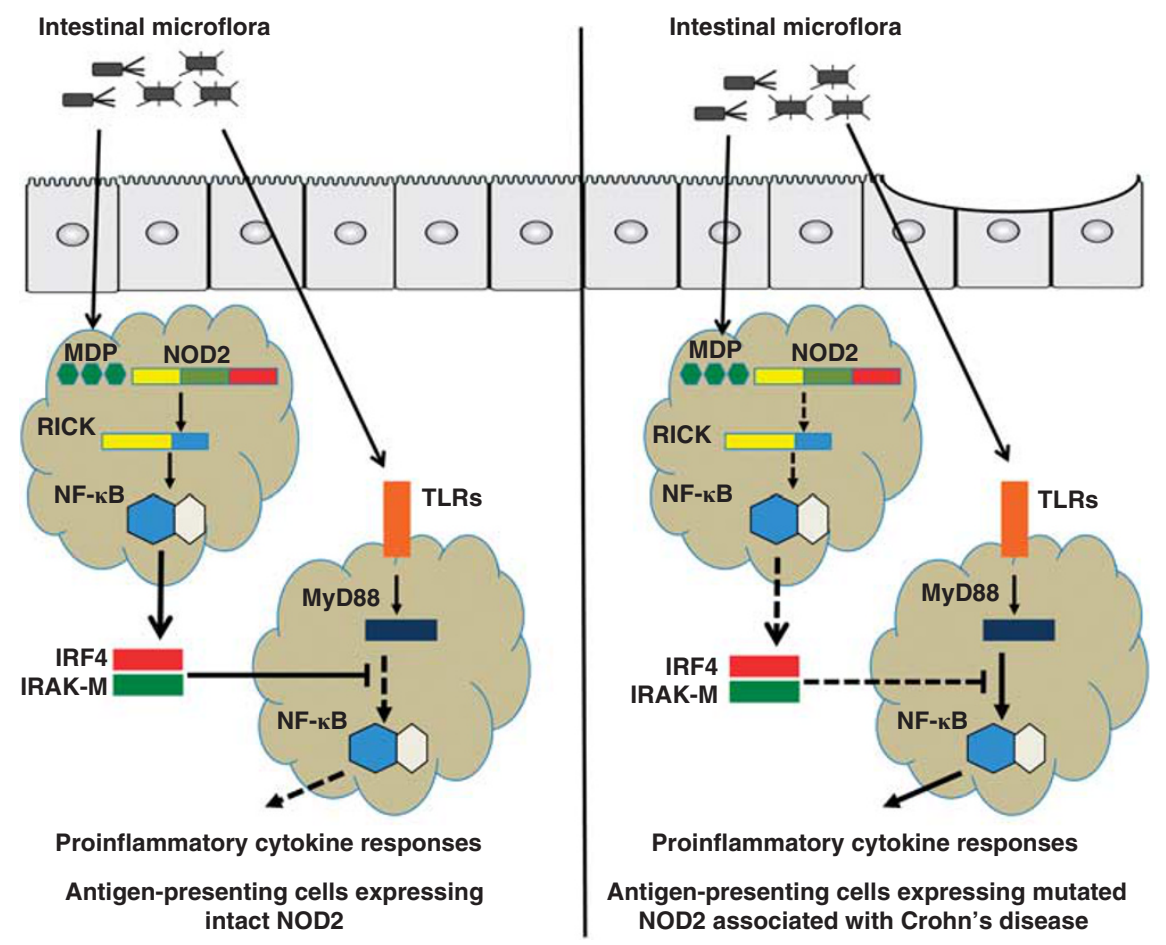

Figure 3 NOD2-regulatory function. MDP-derived from intestinal microflora activates NOD2 in antigen-presenting cells to induce expression of one or more negative regulators of TLR signaling pathways, such as IRF4 and IRAK-M. The latter then suppress NF- $\mathrm{kB}$ activation through TLRs and thus inhibit proinflammatory cytokine responses to intestinal microflora during colonic inflammation. Thus, Crohn's disease-associated NOD2 polymorphisms can contribute to intestinal inflammation by leading to impaired NOD2-induced suppression of TLR signals. The function of this regulatory function is particularly important in the GI tract due to the abundance of the TLR ligands at this site. GI, gastrointestinal tract; MDP, muramyl dipeptide; NOD2, nucleotide-binding oligomerization domain 2; TLR, Toll-like receptor.

bearing on NOD2 with Crohn's disease-like polymorphisms, it was shown that NOD2-deficient mice administered a plasmid expressing an intact NOD2 was protected from colitis induction, whereas the same mice administered a plasmid expressing a Crohn's disease-like LRR frameshift mutation was not protected from colitis induction. ${ }^{45}$ It should be emphasized that the regulatory function of NOD2 could also be demonstrated in a GvHD model, in which it was shown that the absence of NOD2 in donor cells led to more severe GvHD-induced inflammation. ${ }^{46}$ The significance of this is that in this situation, the anti-inflammatory effect of NOD2 can be demonstrated under sterile conditions in which the confounding effects of the gut flora is not operative.

Studies of humans with Crohn's disease with and without NOD2 polymorphisms have also provided data supporting the idea that these polymorphisms lead to loss of NOD2-regulatory function. Here, it was shown that, as discussed above, prolonged stimulation of cells with intact NOD2 leads to diminished secondary responds to TLR or IL-1 $\beta$ stimulation, and stimulation of cells with NOD2 polymorphisms do not result in any reduction in secondary responses. ${ }^{42}$ Thus, although the mechanism of the regulatory response (or cross-tolerizing response) in humans and mice appear to be different (see discussion above), the basic regulatory effect is identical.

Finally, detailed analyses of mice with NOD2 deficiency have provided additional information about NOD2 function that supports the idea that a defect in NOD2 regulation underlies the role of NOD2 in Crohn's disease. In one set of studies, it was shown that in mice with NOD2 deficiency, Peyer's patches display increased permeability to dextran and increased translocation of bacteria. This could be traced to induction of TLRinduced Th1 cytokine responses which increase expression of myosin light-chain kinase, an enzyme previously shown to influence epithelial barrier function by its effects of tight junction proteins. ${ }^{76,77}$ Similar TLR-induced changes in permeability were observed in NOD2-intact mice, but in this case this could be inhibited by provision of exogenous MDP. These data thus suggest that in the absence of NOD2-regulatory effects, the mucosal immune system mounts proinflammatory responses that are subjected to positive feedback by increased translocation of bacteria. In another set of studies already described above, it was shown that NOD2-deficient mice injected with OVA peptide-specific $T$ cells develop colitis when challenged with recombinant $E$. coli expressing OVA peptide but not with $E$. coli expressing an irrelevant antigen. ${ }^{43}$ This model thus again demonstrates that the absence of NOD2 leads to defective regulation of innate responses which then manifest as enhanced antigenspecific responses against gut antigens associated with bacteria. Of interest, in this case, mice were deficient in TLR2 and in NOD2, suggesting that the regulatory function of NOD2 in the GI tract apply particularly to TLR2 ligands. This is not surprising in view of the massive peptidoglycan burden associated with 
the colonic bacterial flora and the potential of peptidoglycan to induce excessive innate responses through TLR2 in the absence of NOD2.

\section{CONCLUSIONS}

Our knowledge of NOD2 function in the past 10 years since it was discovered has disclosed ever deepening complexity as we learn more about the many interactions between NOD2 and other intracellular components. It is now apparent that NOD2 is a major host defense factor, especially with respect to intracellular infections, but at the same time has important regulatory functions, particularly in the GI tract where it is stimulated by host commensal microflora components. It is this latter aspect of NOD2 function that explains the seemingly paradoxical role NOD2 polymorphisms have in the causation of Crohn's disease.

\section{DISCLOSURE}

The authors declared no conflict of interest.

(O) 2011 Society for Mucosal Immunology

\section{REFERENCES}

1. Strober, W., Murray, P.J., Kitani, A. \& Watanabe, T. Signalling pathways and molecular interactions of NOD1 and NOD2. Nat. Rev. Immunol. 6 , 9-20 (2006).

2. Inohara, Chamaillard, McDonald, C. \& Nunez, G. NOD-LRR proteins: role in host-microbial interactions and inflammatory disease. Annu. Rev. Biochem. 74, 355-383 (2005).

3. Cho, J.H. The genetics and immunopathogenesis of inflammatory bowel disease. Nat. Rev. Immunol. 8, 458-466 (2008).

4. Holler, E. et al. Both donor and recipient NOD2/CARD15 mutations associate with transplant-related mortality and GVHD following allogeneic stem cell transplantation. Blood 104, 889-894 (2004).

5. Henckaerts, L. \& Vermeire, S. NOD2/CARD15 disease associations other than Crohn's disease. Inflamm. Bowel Dis. 13, 235-241 (2007).

6. Kobayashi, K.S. et al. Nod2-dependent regulation of innate and adaptive immunity in the intestinal tract. Science 307, 731-734 (2005).

7. Park, J.H. et al. RICK/RIP2 mediates innate immune responses induced through Nod1 and Nod2 but not TLRs. J. Immunol. 178, 2380-2386 (2007).

8. Hasegawa, M. et al. A critical role of RICK/RIP2 polyubiquitination in Nod-induced NF-kappaB activation. EMBO J. 27, 373-383 (2008).

9. Abbott, D.W., Wilkins, A., Asara, J.M. \& Cantley, L.C. The Crohn's disease protein, NOD2, requires RIP2 in order to induce ubiquitinylation of a novel site on NEMO. Curr. Biol. 14, 2217-2227 (2004).

10. Abbott, D.W., Yang, Y., Hutti, J.E., Madhavarapu, S., Kelliher, M.A. \& Cantley, L.C. Coordinated regulation of Toll-like receptor and NOD2 signaling by K63-linked polyubiquitin chains. Mol. Cell. Biol. 27, 6012-6025 (2007)

11. Bertrand, M.J., Doiron, K., Labbe, K., Korneluk, R.G., Barker, P.A. \& Saleh, M. Cellular inhibitors of apoptosis cIAP1 and cIAP2 are required for innate immunity signaling by the pattern recognition receptors NOD1 and NOD2. Immunity 30, 789-801 (2009).

12. Kim, J.Y., Omori, E., Matsumoto, K., Nunez, G. \& Ninomiya-Tsuji, J. TAK1 is a central mediator of NOD2 signaling in epidermal cells. J. Biol. Chem. 283, 137-144 (2008)

13. Tao, M., Scacheri, P.C., Marinis, J.M., Harhaj, E.W., Matesic, L.E. \& Abbott, D.W. ITCH K63-ubiquitinates the NOD2 binding protein, RIP2, to influence inflammatory signaling pathways. Curr. Biol. 19, 1255-1263 (2009).

14. Tigno-Aranjuez, J.T., Asara, J.M. \& Abbott, D.W. Inhibition of RIP2's tyrosine kinase activity limits NOD2-driven cytokine responses. Genes Dev. 24, 2666-2677 (2010).

15. Windheim, M., Lang, C., Peggie, M., Plater, L.A. \& Cohen, P. Molecular mechanisms involved in the regulation of cytokine production by muramyl dipeptide. Biochem. J. 404, 179-190 (2007).
16. Nembrini, C. et al. The kinase activity of Rip2 determines its stability and consequently Nod1 - and Nod2-mediated immune responses. J. Biol. Chem. 284, 19183-19188 (2009).

17. Clark, N.M., Marinis, J.M., Cobb, B.A. \& Abbott, D.W. MEKK4 sequesters RIP2 to dictate NOD2 signal specificity. Curr. Biol. 18, 1402-1408 (2008)

18. Barnich, N., Aguirre, J.E., Reinecker, H.C., Xavier, R. \& Podolsky, D.K. Membrane recruitment of NOD2 in intestinal epithelial cells is essential for nuclear factor-\{kappa\}B activation in muramyl dipeptide recognition. J. Cell. Biol. 170, 21-26 (2005).

19. Eitel, J. et al. Beta-PIX and Rac1 GTPase mediate trafficking and negative regulation of NOD2. J. Immunol. 181, 2664-2671 (2008).

20. Sabbah, A. et al. Activation of innate immune antiviral responses by Nod2. Nat. Immunol. 10, 1073-1080 (2009).

21. Watanabe, T. et al. NOD1 contributes to mouse host defense against Helicobacter pylori via induction of type I IFN and activation of the ISGF3 signaling pathway. J. Clin. Invest. 120, 1645-1662 (2010).

22. McDonald, C. et al. A role for Erbin in the regulation of Nod2-dependent NF-kappaB signaling. J. Biol. Chem. 280, 40301-40309 (2005).

23. Yamamoto-Furusho, J.K., Barnich, N., Xavier, R., Hisamatsu, T. \& Podolsky, D.K. Centaurin beta1 down-regulates nucleotide-binding oligomerization domains 1- and 2-dependent NF-kappaB activation. J. Biol. Chem. 281, 36060-36070 (2006).

24. Bielig, H. et al. A function for AAMP in Nod2-mediated NF-kappaB activation. Mol. Immunol. 46, 2647-2654 (2009).

25. Hsu, Y.M. et al. The adaptor protein CARD9 is required for innate immune responses to intracellular pathogens. Nat. Immunol. 8, 198-205 (2007).

26. Yeretssian, G. et al. Non-apoptotic role of BID in inflammation and innate immunity. Nature 474, 96-99 (2011).

27. Hasegawa, M. et al. Mechanism of ASC-mediated apoptosis: biddependent apoptosis in type Il cells. Oncogene 26, 1748-1756 (2007).

28. Mizushima, N., Levine, B., Cuervo, A.M. \& Klionsky, D.J. Autophagy fights disease through cellular self-digestion. Nature 451, 1069-1075 (2008).

29. Travassos, L.H. et al. Nod1 and Nod2 direct autophagy by recruiting ATG16L1 to the plasma membrane at the site of bacterial entry. Nat Immunol. 11, 55-62 (2010).

30. Cooney, R. et al. NOD2 stimulation induces autophagy in dendritic cells influencing bacterial handling and antigen presentation. Nat. Med. 16, 90-97 (2010).

31. Deretic, V. Multiple regulatory and effector roles of autophagy in immunity. Curr. Opin. Immunol. 21, 53-62 (2009).

32. Watanabe, T., Kitani, A., Murray, P.J. \& Strober, W. NOD2 is a negative regulator of Toll-like receptor 2-mediated T helper type 1 responses. Nat. Immunol. 5, 800-808 (2004).

33. Fritz, J.H. et al. Synergistic stimulation of human monocytes and dendritic cells by Toll-like receptor 4 and NOD1- and NOD2-activating agonists. Eur. J. Immunol. 35, 2459-2470 (2005).

34. Hirata, N. et al. Selective synergy in anti-inflammatory cytokine production upon cooperated signaling via TLR4 and TLR2 in murine conventional dendritic cells. Mol. Immunol. 45, 2734-2742 (2008).

35. Leber, J.H., Crimmins, G.T., Raghavan, S., Meyer-Morse, N.P., Cox, J.S. \& Portnoy, D.A. Distinct TLR- and NLR-mediated transcriptional responses to an intracellular pathogen. PLoS Pathog. 4, e6 (2008).

36. Kim, Y.G., Park, J.H., Shaw, M.H., Franchi, L., Inohara, N. \& Nunez, G. The cytosolic sensors Nod1 and Nod2 are critical for bacterial recognition and host defense after exposure to Toll-like receptor ligands. Immunity $\mathbf{2 8 ,}$ 246-257 (2008).

37. Hsu, L.C. et al. A NOD2-NALP1 complex mediates caspase-1-dependent IL-1 beta secretion in response to Bacillus anthracis infection and muramyl dipeptide. Proc. Natl Acad. Sci. USA 105, 7803-7808 (2008).

38. Pan, Q. et al. MDP-induced interleukin-1 beta processing requires Nod2 and CIAS1/NALP3. J. Leukoc. Biol. 82, 177-183 (2007).

39. Toma, C. et al. Pathogenic Vibrio activate NLRP3 inflammasome via cytotoxins and TLR/nucleotide-binding oligomerization domain-mediated NF-kappa B signaling. J. Immunol. 184, 5287-5297 (2010).

40. Kullberg, B.J. et al. Crohn's disease patients homozygous for the 3020insC NOD2 mutation have a defective NOD2/TLR4 cross-tolerance to intestinal stimuli. Immunology 123, 600-605 (2008).

41. Hedl, M., Li, J., Cho, J.H. \& Abraham, C. Chronic stimulation of Nod2 mediates tolerance to bacterial products. Proc. Natl Acad. Sci. USA 104, 19440-19445 (2007) 
42. Hedl, M. \& Abraham, C. Secretory mediators regulate Nod2-induced tolerance in human macrophages. Gastroenterology 140, 231-241 (2010).

43. Watanabe, T., Kitani, A., Murray, P.J., Wakatsuki, Y., Fuss, I.J. \& Strober, W. Nucleotide binding oligomerization domain 2 deficiency leads to dysregulated TLR2 signaling and induction of antigen-specific colitis. Immunity 25, 473-485 (2006).

44. Yang, Z. et al. NOD2 transgenic mice exhibit enhanced MDP-mediated down-regulation of TLR2 responses and resistance to colitis induction. Gastroenterology 133, 1510-1521 (2007).

45. Watanabe, T. et al. Muramyl dipeptide activation of nucleotide-binding oligomerization domain 2 protects mice from experimental colitis. J. Clin. Invest. 118, 545-559 (2008).

46. Penack, O. et al. NOD2 regulates hematopoietic cell function during graftvs.-host disease. J. Exp. Med. 206, 2101-2110 (2009).

47. Negishi, H. et al. Negative regulation of Toll-like-receptor signaling by IRF-4. Proc. Natl Acad. Sci. USA 102, 15989-15994 (2005).

48. Kobayashi, K., Hernandez, L.D., Galan, J.E., Janeway, C.A. Jr, Medzhitov, R. \& Flavell, R.A. IRAK-M is a negative regulator of Toll-like receptor signaling. Cell 110, 191-202 (2002).

49. Ogura, Y. et al. Expression of NOD2 in Paneth cells: a possible link to Crohn's ileitis. Gut 52, 1591-1597 (2003).

50. Biswas, A. et al. Induction and rescue of Nod2-dependent Th1-driven granulomatous inflammation of the ileum. Proc. Natl Acad. Sci. USA 107, 14739-14744 (2010).

51. Hruz, P. et al. NOD2 contributes to cutaneous defense against Staphylococcus aureus through alpha-toxin-dependent innate immune activation. Proc. Natl Acad. Sci. USA 106, 12873-12878 (2009).

52. Shimada, K. et al. The NOD/RIP2 pathway is essential for host defenses against Chlamydophila pneumoniae lung infection. PLoS Pathog. 5, e1000379 (2009).

53. Shaw, M.H. et al. T cell-intrinsic role of Nod2 in promoting type 1 immunity to Toxoplasma gondii. Nat. Immunol. 10, 1267-1274 (2009).

54. Ferwerda, G. et al. NOD2 and toll-like receptors are nonredundant recognition systems of Mycobacterium tuberculosis. PLoS Pathog. 1, 279-285 (2005).

55. Coulombe, F. et al. Increased NOD2-mediated recognition of N-glycolyl muramyl dipeptide. J. Exp. Med. 206, 1709-1716 (2009).

56. Pandey, A.K. et al. NOD2, RIP2 and IRF5 play a critical role in the type I interferon response to Mycobacterium tuberculosis. PLoS Pathog. 5 , e1000500 (2009).

57. Divangahi, M. et al. NOD2-deficient mice have impaired resistance to Mycobacterium tuberculosis infection through defective innate and adaptive immunity. J. Immunol. 181, 7157-7165 (2008).

58. Gandotra, S., Jang, S., Murray, P.J., Salgame, P. \& Ehrt, S. Nucleotidebinding oligomerization domain protein 2-deficient mice control infection with Mycobacterium tuberculosis. Infect. Immun. 75, 5127-5134 (2007).

59. Petnicki-Ocwieja, T. et al. Nod2 suppresses Borrelia burgdorferi mediated murine Lyme arthritis and carditis through the induction of tolerance. PLOS One. 6, e17414 (2010).
60. Dugan, J.W. et al. Nucleotide oligomerization domain-2 interacts with 2 '-5'-oligoadenylate synthetase type 2 and enhances RNase- $L$ function in THP-1 cells. Mol. Immunol. 47, 560-566 (2009).

61. Magalhaes, J.G. et al. Nod2-dependent Th2 polarization of antigenspecific immunity. J. Immunol. 181, 7925-7935 (2008).

62. Fritz, J.H. et al. Nod1-mediated innate immune recognition of peptidoglycan contributes to the onset of adaptive immunity. Immunity 26, 445-459 (2007).

63. van Beelen, A.J. et al. Stimulation of the intracellular bacterial sensor NOD2 programs dendritic cells to promote interleukin-17 production in human memory T cells. Immunity 27, 660-669 (2007).

64. Strober, W., Fuss, I. \& Mannon, P. The fundamental basis of inflammatory bowel disease. J. Clin. Invest. 117, 514-521 (2007).

65. Wehkamp, J. et al. NOD2 (CARD15) mutations in Crohn's disease are associated with diminished mucosal alpha-defensin expression. Gut 53, 1658-1664 (2004).

66. Strober, W. The multifaceted influence of the mucosal microflora on mucosal dendritic cell responses. Immunity 31, 377-388 (2009).

67. Boirivant, M. et al. A transient breach in the epithelial barrier leads to regulatory T-cell generation and resistance to experimental colitis. Gastroenterology 135, 1612-1623.e5 (2008).

68. Strober, W. Adherent-invasive E. coli in Crohn disease: bacterial "agent provocateur". J. Clin. Invest. 121, 841-844 (2011).

69. Chassaing, B. et al. Crohn disease-associated adherent-invasive E. coli bacteria target mouse and human Peyer's patches via long polar fimbriae. J. Clin. Invest. 121, 966-975 (2011).

70. Garrett, W.S. et al. Communicable ulcerative colitis induced by T-bet deficiency in the innate immune system. Cell 131, 33-45 (2007).

71. Wehkamp, J. et al. The Paneth cell alpha-defensin deficiency of ileal Crohn's disease is linked to Wnt/Tcf-4. J. Immunol. 179, 3109-3118 (2007).

72. Simms, L.A., Doecke, J.D., Walsh, M.D., Huang, N., Fowler, E.V. \& Radford-Smith, G.L. Reduced alpha-defensin expression is associated with inflammation and not NOD2 mutation status in ileal Crohn's disease. Gut 57, 903-910 (2008).

73. Noguchi, E., Homma, Y., Kang, X., Netea, M.G. \& Ma, X. A Crohn's disease-associated NOD2 mutation suppresses transcription of human IL10 by inhibiting activity of the nuclear ribonucleoprotein hnRNP-A1. Nat. Immunol. 10, 471-479 (2009).

74. Maeda, S. et al. Nod2 mutation in Crohn's disease potentiates NF-kappaB activity and IL-1beta processing. Science $307,734-738$ (2005).

75. Ferwerda, G. et al. Engagement of NOD2 has a dual effect on prolL-1 beta mRNA transcription and secretion of bioactive IL-1beta. Eur. J. Immunol. 38, 184-191 (2008).

76. Barreau, F. et al. CARD15/NOD2 is required for Peyer's patches homeostasis in mice. PLoS One 2, e523 (2007).

77. Barreau, F. et al. Nod2 regulates the host response towards microflora by modulating T cell function and epithelial permeability in mouse Peyer's patches. Gut 59, 207-217 (2010). 